\title{
Phosphorylation of SMC1 by ATR is required for desferrioxamine (DFO)-induced apoptosis
}

\author{
EY So ${ }^{1}$, M Ausman $^{1}$, T Saeki ${ }^{2}$ and T Ouchi ${ }^{* 1}$
}

DNA damage signaling pathways are initiated in response to chemical reagents and radiation damage, as well as in response to hypoxia. It is implicated that structural maintenance of chromosomes 1 (SMC1) is not only a component of the cohesion complex but also facilitates the activation of DNA damage checkpoint proteins. Here, we studied the mechanism of DNA damage checkpoint activated by ATR-SMC1 pathway when cells are treated with desferrioxamine (DFO), a hypoxia-mimetic reagent. We show that DFO treatment induces phosphorylation of SMC1 at Ser966, NBS1 at Ser343, Chk1 at Ser317, Chk2 at Thr68, and p53 at Ser15. Among these sites, phosphorylation of SMC1, NBS1, and Chk1 by DFO are mediated by ATR as it is greatly reduced in both ATR-deficient human fibroblasts and HCT116 human colon cancer cells in which ATR is heterozygously mutated, whereas these proteins are phosphorylated in cells deficient for ATM and DNA-PKcs. DFO-induced apoptosis is decreased in ATR-mutant HCT116 cells, although p53 is normally activated in those cells. Expression of SMC1 S966A in which Ser966 is substituted to Ala attenuates apoptosis and phosphorylation of Chk1 at Ser317 after DFO treatment, although levels of HIF1 $\alpha$ are not significantly changed. These results suggest that DFO induces apoptosis through the ATR-SMC1 arm of the pathway.

Cell Death and Disease (2011) 2, e128; doi:10.1038/cddis.2011.9; published online 10 March 2011

Subject Category: Cancer

Cellular DNA is continuously exposed to both external and internal DNA insults such as UV- or ionizing radiation (IR), chemicals, and oxidative stress. An appropriate response to these DNA damage is essential for maintenance of genome stability whose dysfunction is causal for cancer development. ${ }^{1}$ Mechanisms of sensing and repairing damaged DNA are conserved among the species, supporting a notion that regulation of this pathway sensing DNA stress is crucial for the normal proliferation of the whole organism. When cells are DNA damaged, sensor molecules in this system detect them and activate the cell cycle checkpoint that induces growth arrest, whereas these damages are repaired by a mechanism such as homologous recombination or non-homologous endjoining. ${ }^{2,3}$ If not repaired properly, cells undergo apoptosis. It is implicated that several protein kinases are involved in this system, including ATM/ATR protein Ser/Thr kinases, and DNA-dependent protein catalytic subunit (DNA-PKcs). ATM, ATR, and DNA-PK belongs to phosphoinositide 3-kinaserelated kinase family and phosphorylates substrates, which are essential to transduce checkpoint signals to downstream effectors, those including the MRN (Mre11/Rad50/NBS1) complex, Fanconi anemia proteins, and the BRCA1 breast cancer tumor-susceptible protein..$^{4,5}$

Hypoxia is commonly and frequently observed in solid tumors and results from the imbalance of oxygen supply and consumption by successive layers of tumor cells because of unregulated cell growth. ${ }^{6}$ Because lesions of hypoxic tumor tissues are resistant to treatment and are associated with a poor clinical prognosis, tumor hypoxia is a main obstacle for therapeutic strategies. Cellular hypoxia results in a rapid reduction in ATP levels, activation of G1 phase cell cycle checkpoint, inhibition of DNA replication, decreased protein synthesis, and increased protein degradation. ${ }^{7}$ Although hypoxia itself seems to be insufficient to induce DNA damage, it can induce genetic instability through resistance to apoptosis and decreased DNA repair in tumor tissue. ${ }^{8}$ Once DNA damage signaling pathways are initiated in response to hypoxia and hypoxia reoxygenation, ATR protein is activated, leading to phosphorylation of $\mathrm{p} 53$ and Chk $1^{9}$ ATM is also required to phosphorylate $p 53$ and activate G2 checkpoint after reoxygenation..$^{10}$ Previous study showed that, in contrast to IR-activated ATM, the hypoxia-induced activation of both ATR and ATM is independent of MRN complex, ${ }^{11}$ indicating that there are alternative mechanisms for activating ATM/ATR under hypoxia condition.

Structural maintenance of chromosomes (SMC) proteins are evolutionarily conserved chromosomal proteins that are components of the cohesion complex, necessary for sister chromatid cohesion. ${ }^{12}$ Also, SMC1, as a subunit of cohesion, is not only activates DNA repair but also facilitates the recruitment of checkpoint proteins, which activates the intra-S and G2/M checkpoints, indicating that the activation of SMC1

\footnotetext{
${ }^{1}$ Department of Medicine, NUHS, Systems Biology Program, Pritzker School of Medicine, The University of Chicago, Evanston, IL, USA and ${ }^{2}$ Department of Breast Oncology, Saitama International Medical Center, Hidaka, Saitama, Japan

*Corresponding author: T Ouchi, Department of Medicine, NUHS, The University of Chicago, 1001 University Place, Evanston, IL 60201, USA. Tel: + 224 364 7687; Fax: + 224364 7402; E-mail: touchi@bsd.uchicago.edu

Keywords: ATR; SMC1; Hypoxia

Abbreviations: SMC1, structural maintenance of chromosomes 1; ATR, ATM-, Rad3-related; DFO, desferrioxamine; ATM, ataxia-telangiectasia mutated; DNA-Pk, DNA-dependent protein kinase; NBS1, Nijmegen breakage syndrome 1; Chk1/2, checkpoint kinase 1/2; HIF1 $\alpha$, hypoxia-inducible factor 1 alpha; IR, ionizing radiation: $\mathrm{HR}$, homologous recombination; NHEJ, non-homologous end-joining; PIKK, phosphoinositide 3-kinase-related kinase; MRN, Mre11/Rad50/NBS1 complex; BRCA1, breast cancer-susceptibility gene 1; HU, hydroxyurea; NCS, neocarzinostatin; 53BP1, p53-binding protein 1

Received 23.11.10; revised 21.1.11; accepted 26.1.11; Edited by V De Laurenzi
} 
after DNA damage stresses is required for the G2/M checkpoint, as well for intra-S checkpoint. ${ }^{13}$ SMC1 is phosphorylated at specific sites in response to IR in an ATM-dependent manner, and it is shown that phosphorylation of S957 and/or S966 of SMC1 is required for activation of the S-phase checkpoint in response to IR. ${ }^{14}$ The same residues are phosphorylated after treatment with UV irradiation or hydroxyurea $(\mathrm{HU})$ in an ATM-independent manner. Recent studies have also described that SMC1, together with other checkpoint signaling proteins, including p53, 53BP1, and Chk1/Chk2, was phosphorylated rapidly by ATR in ATMdeficient cell lines, ${ }^{15}$ indicating that phosphorylation of SMC1, under conditions of DNA stress, has a crucial role in DNA damage response and subsequent activation of cell cycle checkpoint. Although hypoxia and reoxygenation after hypoxia also induce phosphorylation of Ser957 of SMC1 in human lymphocyte, ${ }^{16}$ the mechanisms and the role of phosphorylated SMC1 in hypoxia remain unclear. Desferrioxamine (DFO) is used to induce hypoxic condition in cell culture, however, it is not well illustrated how it could activate DNA damage pathway. In the present studies, by taking advantage of a subset of specific mutant cell lines, we detailed how DFO activates DNA damage signaling. We describe that DFO-induced phosphorylation of SMC1 at Ser966 and Chk1 at Ser317 was dependent on ATR protein kinase, whereas $\mathrm{HU}$ - or neocarzinostatin (NCS)-induced phosphorylation of SMC1 and Chk1 was not abrogated in ATR-mutant cells. We show that expression of a phospho-deficient form of SMC1, SMC1S966A, can function as a dominant-negative molecule in DFO-induced apoptosis. These results suggest that phosphorylation of SMC1 at Ser966 by ATR has an essential role in inducing apoptosis under hypoxia conditions.

\section{Results}

DFO treatment induces phosphorylation of SMC1. It has previously been demonstrated that hypoxia condition generated by DFO, a hypoxia-mimetic and antioxidant agent, could induce p53-dependent checkpoint signaling in human peripheral blood lymphocytes. ${ }^{17} \mathrm{We}$ started to detail how DFO activates DNA damage pathway. Human colorectal carcinoma HCT116 cells were treated with DFO at different concentrations for $24 \mathrm{~h}$, followed by treatment with either IR-mimetic agent NCS that can produce double-stranded breaks, ${ }^{4}$ or DNA replication inhibitor $\mathrm{HU}$ for $30 \mathrm{~min}$ or $1 \mathrm{~h}$ (Figures 1a and b). Cells were collected and total lysates were subjected to western blotting with antibodies for DNA damage-responsive proteins. It is well illustrated that phosphorylation of ATM Ser1981 is a readout of its activation. ${ }^{18}$ NCS treatment weakly induced phosphorylation of ATM at Ser1981 in HCT116 cells (Figure 1a, lanes 2 and 3 ), but no significant phosphorylation was detected in DFO- and HU-treated HCT116 cells (Figure 1b). Phosphorylation of NBS1at Ser343 was also weakly induced by DFO and NCS (Figure 1a), but no additive or enhanced phosphorylation was observed by DFO plus either NCS or HU (Figures 1a and b). Phosphorylation of Chk2 at Thr68 was weakly induced by higher concentration of DFO (300 and $500 \mu \mathrm{M}$ ) after long exposure of autoradiography, but it was much less than that induced by NCS treatment (Figure 1a). This Chk2 phosphorylation was also induced by $\mathrm{HU}$ alone, but it was similar to the levels those induced by DFO treatment of high concentration (300 and $500 \mu \mathrm{M}$ ) (Figure 1b).

Among the DNA damage-associated proteins studied, SMC1 was strongly phosphorylated at Ser966 by DFO, NCS, and $\mathrm{HU}$ treatment. Of note, ATM's activation is detected only with NCS treatment, suggesting that ATM is not involved in phosphorylation of SMC1 when cells are treated with DFO or HU. Supporting this notion, it is shown that Ser966 of SMC1 is phosphorylated by $\mathrm{HU}$ treatment through ATM-independent manner, whereas IR-induced phosphorylation at this site requires ATM and its substrates, such as NBS1 and BRCA1. ${ }^{14}$ Together these results suggest that phosphorylation of SMC1 by DFO and HU does not require activation of ATM.

ATR is required for DFO-induced phosphorylation of SMC1. Previous reports have shown that ATM and ATR phosphorylate similar motifs of peptides under different conditions of DNA stress. ${ }^{19}$ Recent works have also demonstrated that ATR is required for phosphorylation of checkpoint proteins, such as p53, SMC1, and Chk1/2, after IR in ATM-deficient cells. ${ }^{15}$ As we showed in Figure 1, phosphorylation of SMC1 at Ser966 by DFO does not require activated ATM. From these observations, we reasoned to identify the kinase that is responsible for phosphorylation of SMC1 at Ser966 when treated with DFO. We used three different human fibroblast cell lines, GM00200 (control, $\operatorname{ATM}(+), \operatorname{ATR}(+))$, GM09607 $(\operatorname{ATM}(-))$, and GM18366 $(\operatorname{ATR}(-))$. When these cells were treated with DFO, phosphorylation of SMC1 at Ser966 was detected in control and ATM-deficient cells, but it was greatly decreased in ATR-deficient cell (Figure 2). Although levels of SMC1 in GM18366 cells were lower than those in other two cell lines, and levels of SMC1 in GM09607 cells were slightly higher than those of GM00200 cells, NCS similarly induced phosphorylation of SMC1 in three of these cell lines, suggesting that this phosphorylation of SMC1 is ATM/ATRindependent, or ATM and ATR compensate individual deficiency. It is shown that phosphorylation of DNA-PKcs at Ser2056 indicates the readout of activation of the catalytic activity. ${ }^{20}$ Although levels of DNA-PKcs among these three cell lines were different, DFO-induced phosphorylation of DNA-PKcs at Ser2056 was detected only in ATM-deficient cells. Phosphorylation of DNA-PKcs at this site in ATRdeficient cells, induced by NCS, was much weaker than those detected in control and ATM-deficient cells.

We further detailed the phosphorylation of SMC1 and other proteins involved in DNA damage pathway using isogenic HCT116 cell lines, those including HCT116-p53(-), -DNAPKcs(-), and ATR(-/flox). ${ }^{21-23}$ In HCT116-ATR(-/flox) cells, one allele of the ATR gene is deleted and the other allele is floxed, resulting in significantly decreased levels of the protein (Figure $3 \mathrm{~b}$ and see Moynahan and Jasin ${ }^{3}$ ). Parental HCT116 cells and their isogenic cell lines were treated with DFO, HU, or NCS for $24 \mathrm{~h}$ (Figures $3 \mathrm{a}-\mathrm{c}$ ). Consistent with the results from human fibroblasts (Figure 2), DFO treatment did not significantly induce phosphorylation of SMC1 at Ser966 in HCT116-ATR(-/flox) cells, even though low levels of ATR are 
a

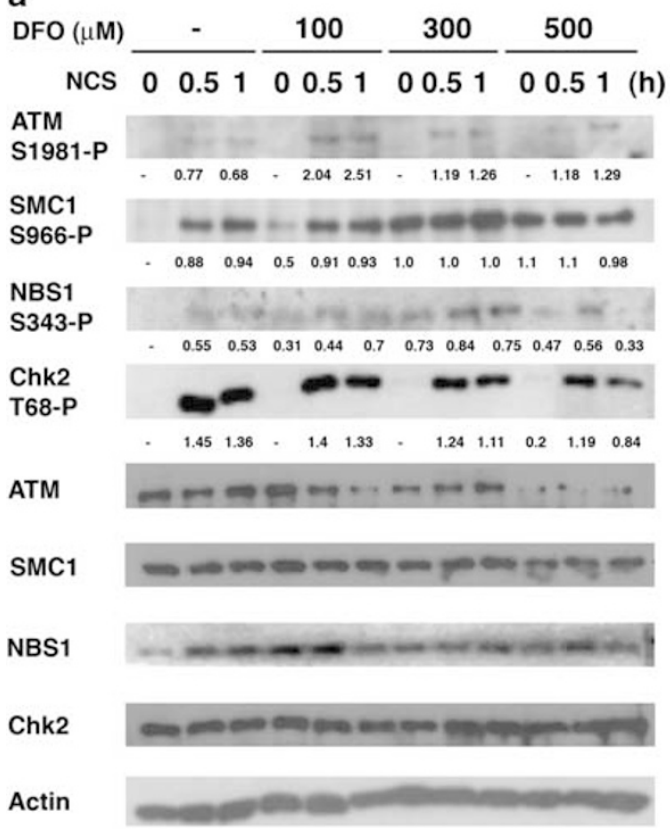

b
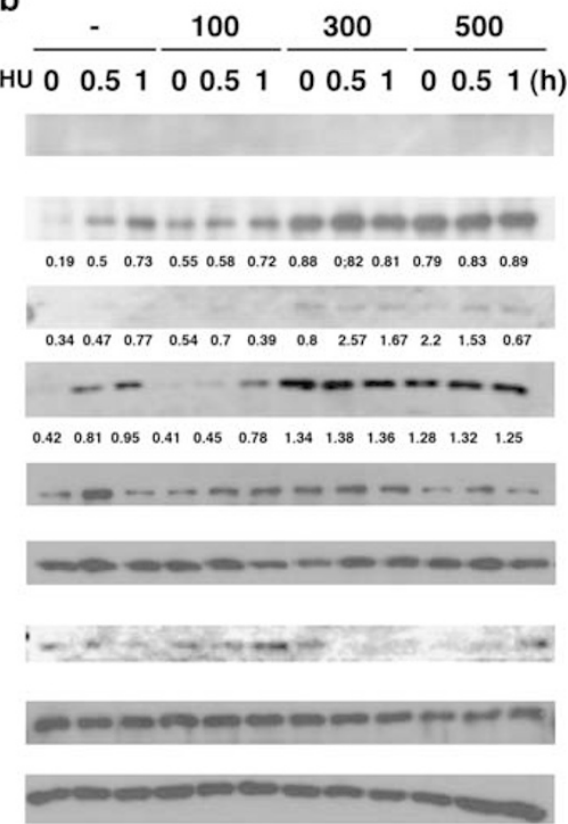

Figure 1 DFO treatment induces phosphorylation of DNA damage response proteins. HCT 116 cells were treated with DFO for $24 \mathrm{~h}$ at indicated concentrations, and then further treated with NCS $(0.5 \mu \mathrm{g} / \mathrm{ml})(\mathbf{a})$ or HU $(3 \mathrm{mM})(\mathbf{b})$ for the indicated times. Total cells extracts were cleared and $20 \mu \mathrm{g}$ of proteins were subjected to a $6 \%$ SDS-PAGE gel. After transferring to PVDF membranes, phosphorylation of indicated proteins was assayed by western blotting with phospho-specific antibodies as indicated. The results of immunoblots were analyzed by densitometry. The numbers under blots show the relative densitometry ratios of phosphoprotein to total protein. Actin protein was used as internal loading control; ND, not detected

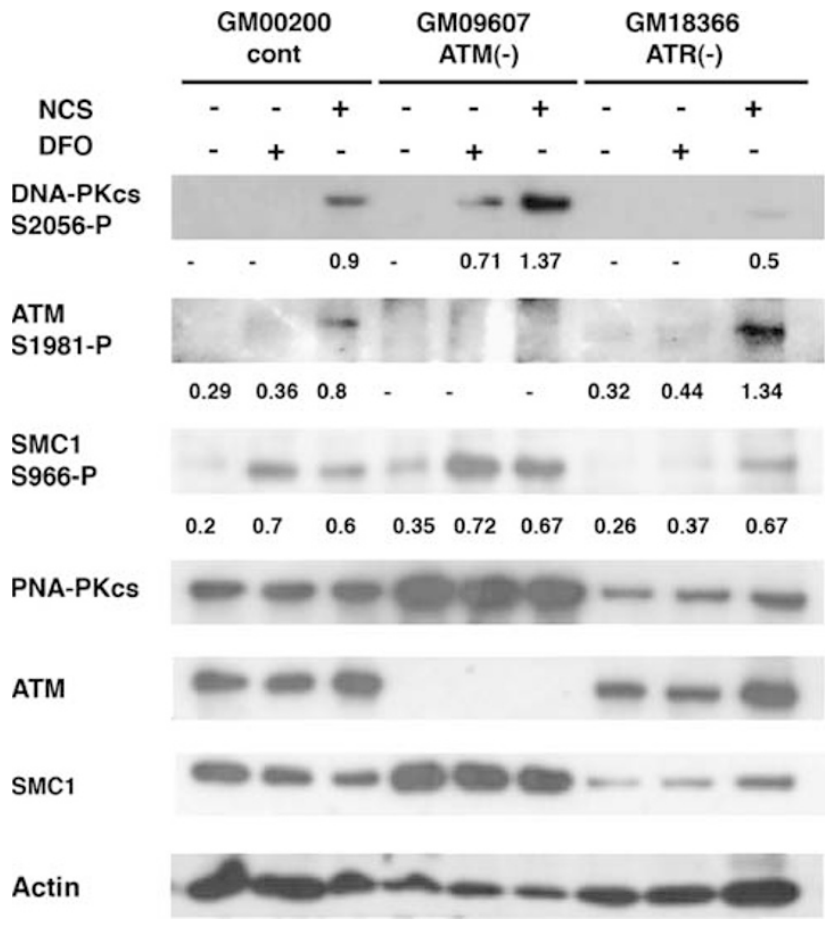

Figure 2 DFO induces phosphorylation of SMC1 is lowered in ATR-deficient fibroblasts. GM00200 (wild type), GM09607 (ATM(-)), and GM18366 (ATR(-)) human fibroblast were treated with NCS $(0.5 \mu \mathrm{g} / \mathrm{ml})$ or DFO $(300 \mu \mathrm{M})$ for $24 \mathrm{~h}$ and assayed by western blotting with anti-phospho antibodies for DNA-PKcs Ser2056, ATM Ser1981, and SMC1 Ser966. Actin protein was a loading control. The level of phosphorylated protein was analyzed by densitometry as described in Figure 1 still expressed in those cells (Figure 3a, lanes 4 and 5, and Figure $3 b$, lanes 7 and 8 ). As shown in previous studies, ${ }^{11}$ we observed reduced level of ATM protein in DNA-PKcs(-) cells compared with parental HCT116 (data not shown). Nevertheless, phosphorylation of SMC1 at Ser966 in HCT116-DNA-PKcs(-) cells was similarly induced to the levels of the parental HCT116 cells in response to these three chemicals (Figures $3 a$ and $b$ ). This phosphorylation of SMC1 induced by NCS was weaker in HCT116-ATR(-/flox) cells, compared with the parental and HCT116-DNA-PKcs $(-)$ cells (Figure 3a, lanes 6 and 9). Interestingly, phosphorylation of Chk2 at Thr68 by DFO was not detected in HCT116-DNAPkcs(-) cells, indicating that DNA-PKcs is essential for this phosphorylation of Chk2 (Figure 3c). Of note, phosphorylation of p53 at Ser 15 and Ser20 is similarly induced by DFO and HU in parental HCT116, HCT116-ATR(-/flox), and HCT116DNA-PKcs(-) cells (Figure $3 b)$. As DFO is a hypoxia-mimetic reagent, treatment of these HCT116 variants with DFO induced HIF1a as previously reported, ${ }^{24}$ although levels of an induced protein are different among these four cell lines.

These results implicate that DFO activates two arms of signaling pathways. Thus, one is to phosphorylate SMC1 at Ser966, NBS1 at Ser343, and Chk1 at Ser317 in an ATRdependent manner, and the other is to phosphorylate Chk2 at The68 in DNA-PKcs-dependent manner.

ATR is necessary for DFO-induced apoptosis. It is shown that anoxia or hypoxia induces a $\mathrm{G} 1$ and intra-S phase arrest. ${ }^{10}$ As DFO is widely used to induce hypoxia condition in cell culture, ${ }^{25}$ we studied the role of ATR in DFO-induced 


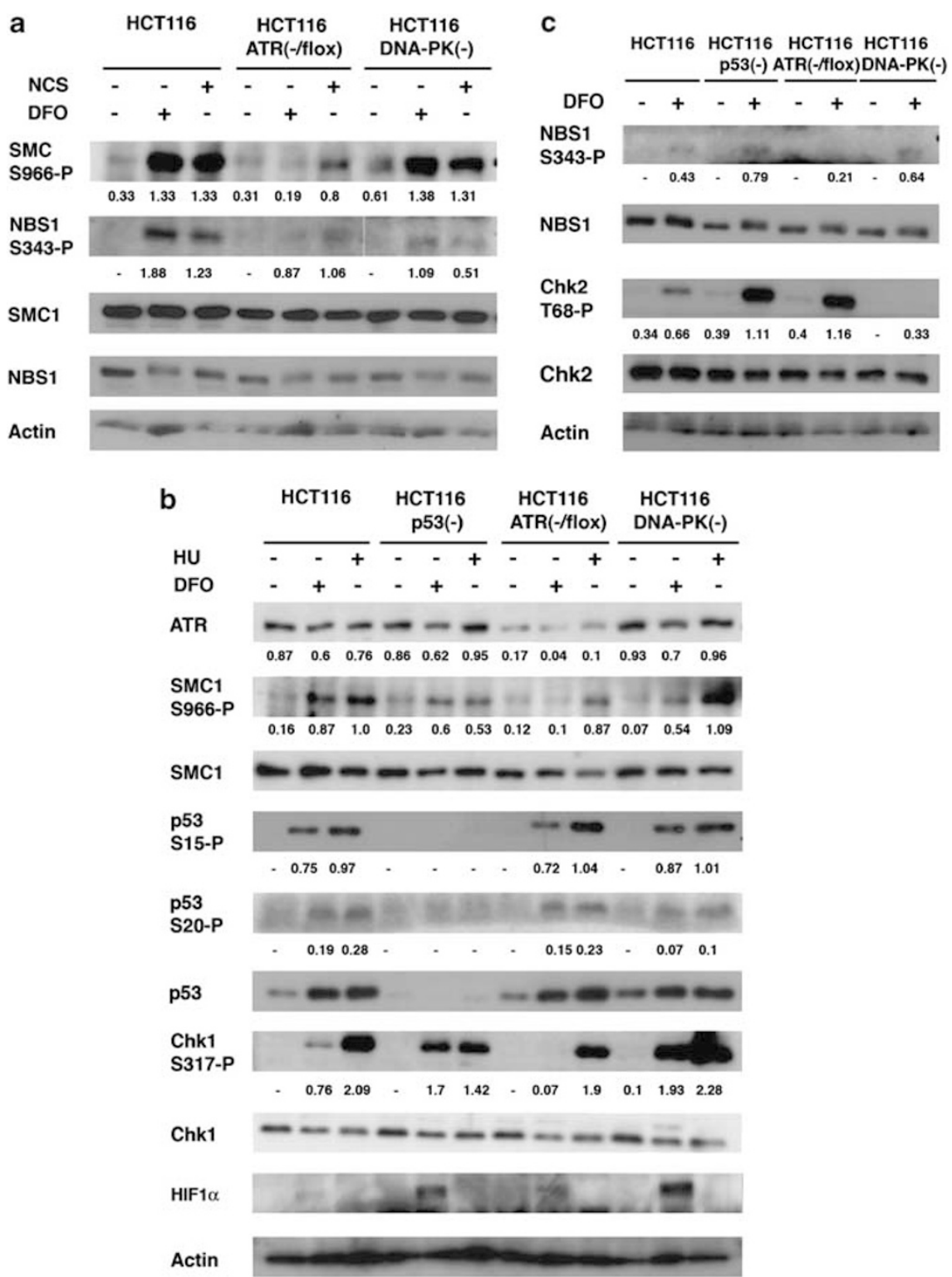

Figure 3 DFO phosphorylates SMC1, NBS1, and Chk1 in ATR-dependent manner. Parental HCT116 (wild type), HCT116-p53(-), HCT116-ATR(-/flox), and HCT116DNA-PKcs(-) cells were treated with DFO $(300 \mu \mathrm{M})$ or NCS $(0.5 \mu \mathrm{g} / \mathrm{ml})$, or HU $(3 \mathrm{mM})$ for $24 \mathrm{~h}$, and then total extracts were studied for western blotting. Phosphorylation of indicated proteins was compared when cells are treated with (a) NCS and DFO, and (b) HU and DFO. (c) Phosphorylation of NBS1 and Chk2 by DFO were compared in isogenic HCT116 cell lines. Actin was used for a loading control. The level of phosphorylation of SMC1, NBS1, p53, Chk1, and Chk2 was expressed by densitometry ratios of phosphoprotein to total protein. The expression of ATR protein in these cells was measured by ratio of ATR to actin protein (b)

cell cycle checkpoint. Parental, HCT116-p53(-), HCT116DNA-PKcs $(-)$, or HCT116-ATR(-/flox) cells were treated with DFO or $\mathrm{HU}$ for $24 \mathrm{~h}$ and collected, then stained with propidium iodide $(\mathrm{PI})$ for cell cycle analysis using flow cytometer (Figure 4).

After DFO treatment, increase in S-phase was most obvious in the parental HCT116 (from 22.4 to $28.8 \%$ ), not in others. Instead, HCT116-p53(-), HCT116-ATR(-/flox), and HCT116-DNA-PKcs(-) cells showed increase in G1 phase. Population in S-phase in HCT116-p53(-), HCT116-ATR (-/flox), and HCT116-DNA-PKcs(-) cells did not change significantly with DFO treatment compared with their individual untreated control, but numbers of cells in G2/M phase were greatly reduced with this treatment.

When cells were treated with $\mathrm{HU}$, all of these cell types showed significant increase in S-phase, although HCT116DNA-PKcs $(-)$ cells showed less accumulation in S-phase (47.4\%) than other cells (HCT116 cells, 55.7\%; HCT116p53(-) cells, 55.1\%; HCT116-ATR(-/flox) cells, 55.2\%).

We further detailed the role of ATR and phosphorylation of SMC1 at Ser966 in regulating apoptosis, in particular. HCT116-ATR(-/flox) cells were transfected with a plasmid expressing Cre-recombinase fused with GFP protein (Cre-GFP) to delete the remaining ATR allele. Cells were 


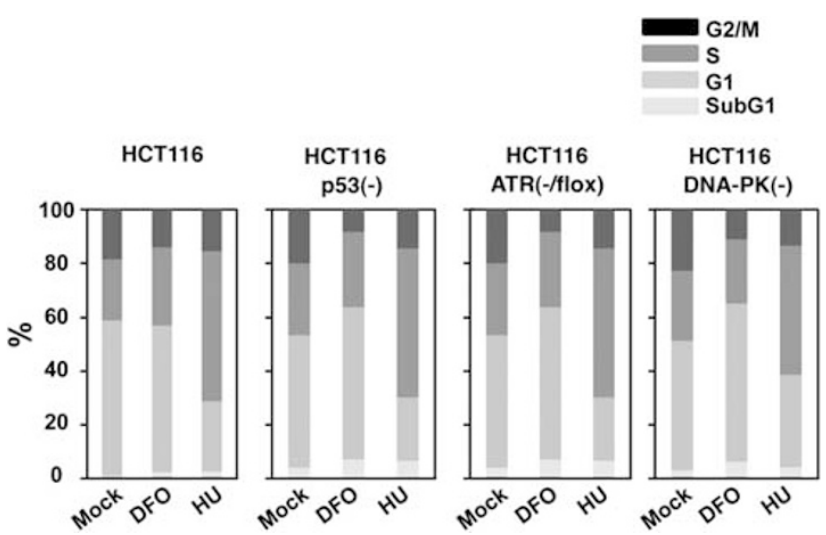

Figure 4 DFO induced cell cycle arrest in wild-type HCT116 cells and isogenic cell lines. Cells were treated with DFO $(300 \mu \mathrm{M})$ or $\mathrm{HU}(3 \mathrm{mM})$ for $24 \mathrm{~h}$. Cell cycle was quantified by single-parameter flow cytometry after PI staining for DNA content. Graphs represent percentages of cells in sub-G1, G1, S, and G1/M phases of cell cycle. Data shown here are representative of three experiments performed

then treated with DFO, NCS, or $\mathrm{HU}$ for $24 \mathrm{~h}$, followed by Annexin $\mathrm{V}$ staining to measure apoptosis (Figure 5a). DFO treatment induces apoptosis in parental HCT116 cells by $\sim 2.5$-fold (from 11.6 to $29.4 \%$ ). Basal levels of apoptosis of Cre-transfected HCT116-ATR(-/flox) are higher (17.8\%) than parental HCT116 cells (11.6\%), indicating that the loss of both the alleles of ATR is intrinsically apoptotic. Level of apoptosis of HCT116-ATR(-/flox) cells was slightly increased $(23.9 \%)$ after DFO treatment ( $\sim 1.3$-fold) (Figure $5 \mathrm{a})$. When Cre-GFP-transfected HCT116 cells were treated with either NCS or HU, induction of apoptosis was 2.4- and $\sim 2.0$-fold, respectively. Levels of apoptosis of Cre-GFP-transfected CT116-ATR(-/flox) cells, treated with NCS and HU, were similar to those of parental HCT116 cells by $\sim 2$.3- and $\sim 2.0$ fold, respectively. These results are schematically indicated in Figure $5 \mathrm{~b}$. These results demonstrate that DFO-induced apoptosis is significantly reduced when ATR is inactivated.

ATR-dependent phosphorylation of SMC1 at S966 is required for DFO-induced apoptosis. On the basis of the results demonstrated in Figure 1, we hypothesized that this ATR-mediated phosphorylation of SMC1is involved in DFOinduced apoptosis that is shown in Figure 5. We explored transient expression of a phospho-deficient mutant form of SMC1 in cell culture. 293T cells and HCT116 cells were transfected with an empty vector or a plasmid expressing a Myc-tagged mutant SMC1 in which Ser966 is substituted by Ala (SMC1S966A) (Figures 6a and b, see Kim et $a l^{14}$ ), and we analyzed apoptotic phenotype after DFO treatment. The expression of mutant SMC1 was confirmed by immunoblotting of anti-Myc antibody. As described in Figure 3, phosphorylation of both Chk1 at Ser317 and NBS1 at Ser343 were induced with DFO treatment in both cell types, but it was weakly decreased in when SMC1S966A was expressed (Figure 6a, lanes 2 and 6; Figure 6b, lanes 2 and 5). It is well illustrated that hypoxia condition induces $\mathrm{HIF} 1 \alpha$, a transcription factor that has an essential role in cellular and systemic responses to hypoxia. ${ }^{25}$ As shown in Figure 6a, DFO induced HIF1 $\alpha$ in 293T cells, and levels of HIF1a were

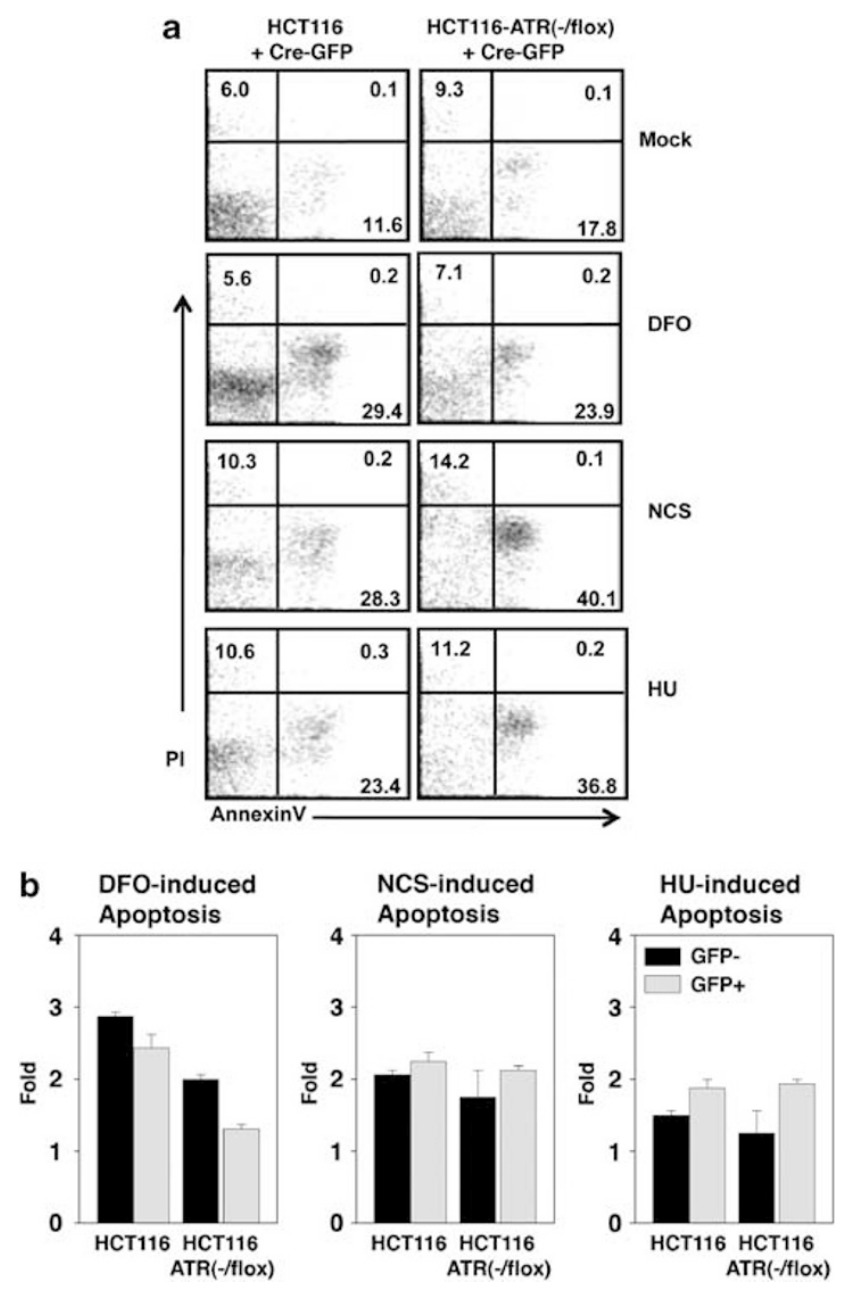

Figure 5 Effect of ATR deficiency on DFO-induced apoptosis. Parental HCT116 and HCT116-ATR(-/flox) cells were transfected with a plsmid expressing Cre-GFP fusion protein or a control vector (not shown). At $48 \mathrm{~h}$ after transfection, cells were treated with DFO $(300 \mu \mathrm{M}), \mathrm{NCS}(0.5 \mu \mathrm{g} / \mathrm{ml})$, or HU $(3 \mathrm{mM})$ for $24 \mathrm{~h}$. Cells were stained with Annexin $\mathrm{V}$ with $\mathrm{PI}$ counter staining. Green fluorescence-positive and -negative cells were gated and separately analyzed using FACSCalibur flow cytometer. (a) Percentage of dot plot represents one of the three independent experiments. (b) Induction of apoptosis of HCT116 and HCT116-ATR(-/flox) cells by DFO, NCS, or HU was indicated as a bar graph. Data were expressed fold induction compared with mock-treated samples

not significantly changed when SMC1 S966A was expressed (Figure 6a, lanes 2 and 6).

To further elucidate the biological roles of SMC1 pathway, we studied whether DFO-induced apoptosis could be modulated by expressing a phospho-mutant form of SMC1, SMC1S966A. After transient transfection of both 293T cells and HCT116 cells with SMC1S966A, cells were treated with DFO, and apoptosis was measured by Annexin V staining. As shown in Figures $6 \mathrm{c}$ and $\mathrm{d}$, expression of SMC1S966A inhibited induction of apoptosis. Inhibition of apoptosis by SMC1S966A in HCT116 cells was not as much as that in 293T cells probably because of lower levels of expression of the protein. To confirm this, levels of Myc-tagged SMC1S966A were compared between HCT116 and 293T cells, and phosphorylation of SMC1 at Ser966 was detected (Figure 6e). As indicated, levels of exogenous SMC1S966A 
were higher in 293T cells, and phosphorylation of SMC1 at Ser966 was sufficiently inhibited in those cells compared with HCT116 cells.

These results indicate that SMC1S966A functions as a dominant-negative protein for DFO-induced apoptosis, and suggest that ATR-mediated phosphorylation of SMC1 at Ser966 is important for this phenotype.

a

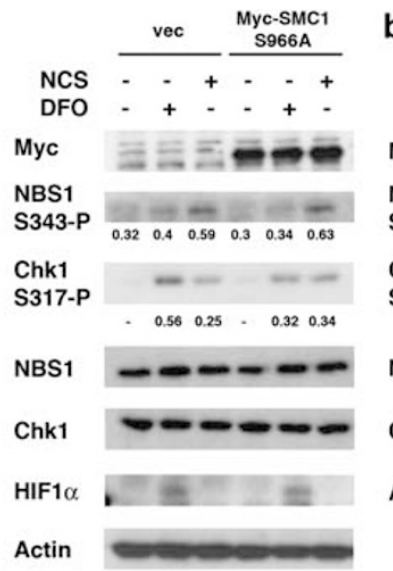

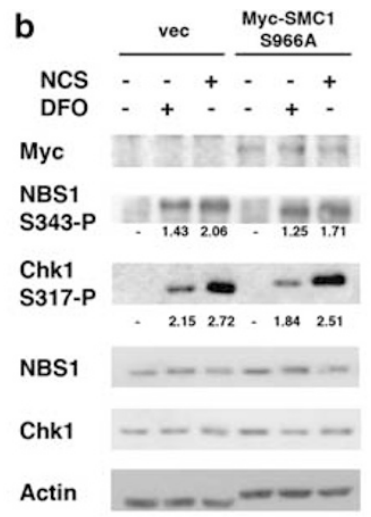

C
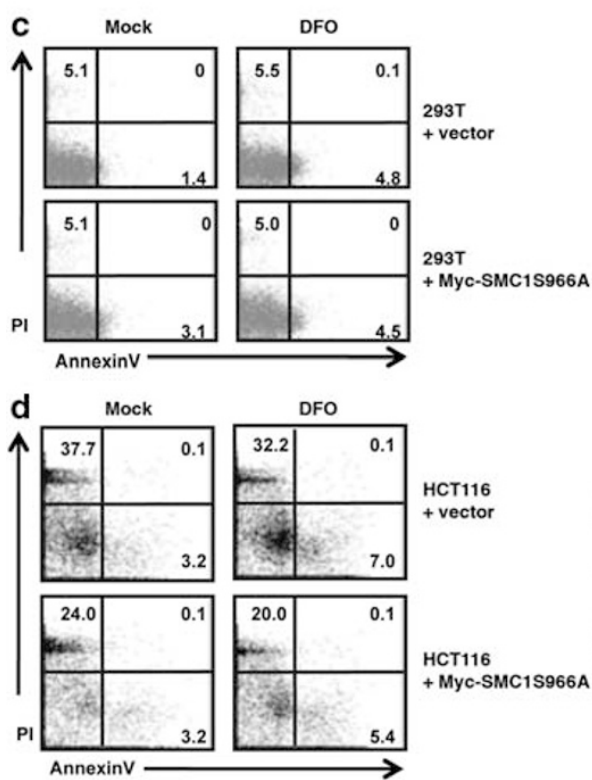

e

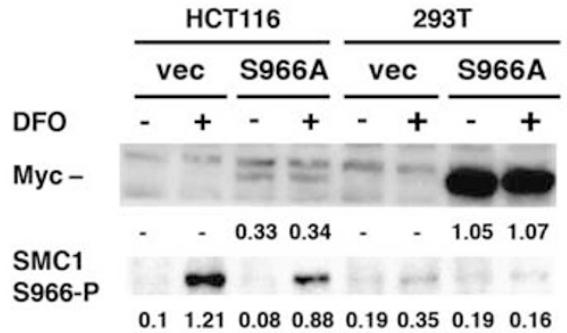

SMC1

Tubulin
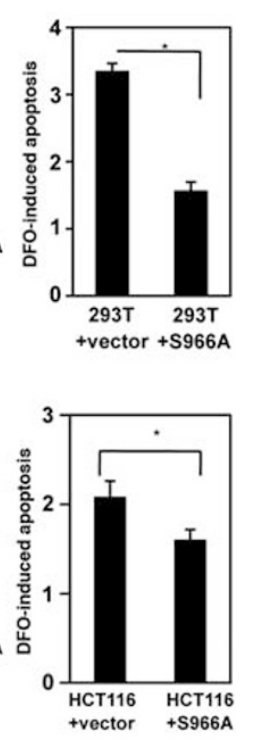

Figure 6 Transfection of 293T cells and HCT116 cells with S966A-mutant SMC1 inhibited the DFO-induced apoptosis. (a) 293T cells and (b) HCT116 cells were transfected with myc-tagged mutant SMC1 plasmid (Myc-SMC1S966A) or a control vector. After $48 \mathrm{~h}$, cells were treated with DFO $(300 \mu \mathrm{M})$ or NCS $(0.5 \mu \mathrm{g} / \mathrm{ml})$ for additional $24 \mathrm{~h}$. Total extracts were studied for western blotting with indicated antibodies to detect level of HIF1 $\alpha$ and phosphorylated proteins of NBS1 S343P and Chk1 at S317P. Actin blot serves as a loading control. (c) 293T cells and (d) HCT116 cells were transfected with plasmid containing mutant SMC1 (MycSMC1S966A) or a control vector. After $48 \mathrm{~h}$, cells were treated with DFO $(300 \mu \mathrm{M})$ for additional $24 \mathrm{~h}$ and then apoptosis was analyzed using a flow cytometer (left panel). Dot plot represents two independent experiments, indicating Annexin $\mathrm{V}$ staining (right panel). DFO-induced apoptosis was expressed as fold-induction compared with untreated samples (right panel, ${ }^{\star} P<0.05$, Student's $t$-test). (e) Levels of Myc-tagged SMC1S966A and phosphorylation of endogenous SMC1 at Ser966 were compared in HCT116 and 293T cell lines. The phosphorylation and expression of proteins were analyzed by densitometry as described in Figure 1 
ATR-deficient fibroblasts, but not in ATM-deficient cells Phosphorylation of SMC1 at Ser966 by NCS was detected in HCT116-ATR(-/flox) cells, although it is slightly weaker than the other cell lines examined. Given that this phosphorylation of SMC1 by DFO is greatly reduced in HCT116-ATR (-/flox) cells, these results implicate that ATR is critical for SMC1's phosphorylation in DFO pathway. Recent studies have illustrated that phosphorylation of SMC1 is required for the activation of S-phase checkpoint after IR, and phosphorylation of NBS1 is essential for this phosphorylation of SMC1. ${ }^{27}$ Our results showed that DFO induces strong phosphorylation of SMC1 at Ser966, although phosphorylation of NBS1 at Ser343 is quite low in wild-type ATR cells (Figure 1). Taken together, we consider that SMC1 can be activated by at least two distinct pathways, one is ATM/NBS1/SMC1 in response to IR and the other is ATR/SMC1 in response to DFO (Figure 7).

Our results demonstrated that phosphorylation of Chk2 at Thr68 is impaired in DFO-treated HCT116-DNA-PKcs $(-)$ cells. Recent studies have shown that inactivation of DNAPKcs by siRNA or kinase inhibitor, Nu7026, results in dysfunction of mitotic checkpoint and attenuation of Chk2 phosphorylation at Thr68 in response to IR. ${ }^{28}$ Together with our results, both DFO and IR may activate DNA-PKcs to induce phosphorylation of Chk2.

In 293T cells expressing SMC1 S966A, phosphorylation of Chk1 at Ser317 was slightly lowered but HIF1 $\alpha$ levels were not changed significantly after DFO treatment compared with control cells. Induction of HIF $1 \alpha$ by DFO was detected in all of the isogenic HCT116 variants examined, although HCT116ATR(-/flox) cells constitutively underwent apoptosis even without DFO treatment. Therefore, it remains to be elucidated whether induction of $\mathrm{HIF} 1 \alpha$ is essential for DFO-induced apoptosis. Thus, it is suggested that DFO induces apoptosis in ATR/Chk1-dependent but not in HIF1 $\alpha$-independent pathway.

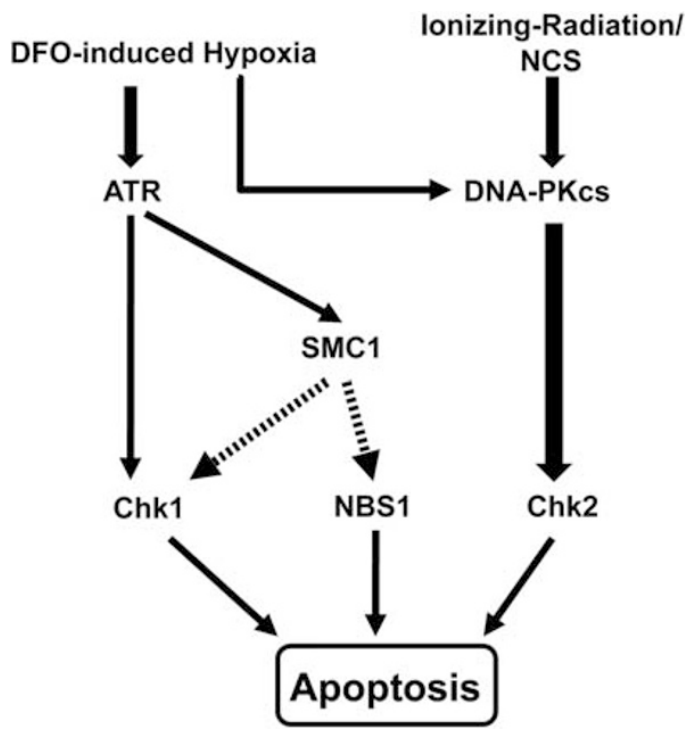

Figure 7 Schematic presentation of pathways indicating SMC1 is involved in DFO-induced apoptosis
It remains to be clear how phosphorylated SMC1 produces apoptotic signals. Although it is well known that the phosphorylation of SMC1 is required for the IR-induced S-phase cell cycle checkpoint ${ }^{14}$ and also associated with G2/M checkpoint as a subunit of cohesion with SMC3, ${ }^{13}$ these studies have not directly addressed how SMC1 regulates apoptosis. As one possible model, it is plausible that SMC1 phosphorylated at Ser966 specifically binds to subsets of cellular proteins, generating proapoptotic complex. This model is raised by recent studies demonstrating that SMC1 phosphorylated at both Ser957 and Ser966 preferentially binds to Rae1, a microtubule-bound RNA export factor 1 , at the mitotic spindle pole. $^{29}$ These studies suggest that phosphorylation status of SMC1 can determine its intermolecular interaction with cellular components. Identification of proteins that preferentially binds to phosphorylated SMC1 is in progress.

\section{Materials and Methods}

Cells, chemicals, and plasmids. GM00200 (control), GM09607 (ATM(-)), GM18366 (ATR(-)) human fibroblast cell lines were obtained from the National Institute of General Medical Sciences Coriell Cell Repositories (Camden, NJ, USA). Parental HCT-116, HCT116-ATR(-/flox) were purchased from ATCC (Manassas, VA, USA), HCT116-p53(-) cells are provided by Dr. B Vogelstein at Johns Hopkins University, and a DNA-PK-mutant HCT116 cell line (HCT116-DNA-PKcs(-)) was kindly provided by Dr. E Hendrickson at University of Minnesota Medical School. All of these cell lines were grown in DMEM media (Invitrogen, Carlsbad, CA, USA) containing 10\% fetal bovine serum (Invitrogen), penicillin/streptomycin and $10 \mathrm{mM}$ sodium pyruvate (Sigma, St. Louis, MO, USA). Desferrioxamine mesylate (DFO) was obtained from Calbiochem (La Jolla, CA, USA). HU and NCS were purchased from Sigma. Myc-tagged wild-type SMC1 and S966A-mutant SMC1 expression vectors were gratefully provided by Dr. M Kastan (St. Jude Children's Research Hospital, Memphis, TN, USA) and pCAG-Cre-GFP expression vector was purchased from Addgene (Cambridge, MA, USA).

Immunoblotting and protein assays. Cells were treated with DFO $(100$, 300 , or $500 \mu \mathrm{M})$ for $24 \mathrm{~h}$, followed by HU $(3 \mathrm{mM})$, or NCS $(0.5 \mu \mathrm{g} / \mathrm{ml})$ for the indicated time, and then lysed in ice-cold lysis buffer $(50 \mathrm{mM}$ Tris- $\mathrm{HCl}(\mathrm{pH} 7.6)$, $150 \mathrm{mM} \mathrm{NaCl}, 1 \mathrm{mM}$ EDTA (pH 8.0), $20 \mathrm{mM} \mathrm{NaF}, 1 \mathrm{mM} \mathrm{Na}_{3} \mathrm{VO}_{4}, 1 \% \mathrm{NP} 40,0.5 \mathrm{mM}$ dithiothreitol) in the presence of protease-inhibitor mix (leupeptin, aprotinin, and PMSF, $10 \mu \mathrm{g} / \mathrm{ml}$, respectively). After centrifugation $(12000 \mathrm{~g}, 10 \mathrm{~min}$ ), soluble supernatants were prepared and protein concentrations were calculated using the Bio-Rad protein assay kit (Bio-Rad, Hercules, CA, USA). Total cell lysate $(20 \mu \mathrm{g})$ was loaded and separated by $6.0 \%$ SDS-polyacrylamide gels. Transfer to a PVDF membrane (Immobilon-P, Millipore, Billerica, MA, USA) was carried out using semidry transfer method (Trans-Blot, Bio-Rad) in $25 \mathrm{mM}$ Tris, $192 \mathrm{mM}$ glycine, and $10 \%$ methanol for $1 \mathrm{~h}$ at $20 \mathrm{~V}$. Membranes were blocked in $5 \%$ non-fat dried milk in Tris-buffered saline $/ 0.1 \%$ Tween-20 and incubated with primary antibodies and Horseradish peroxidase-conjugated secondary antibodies (Santa Cruz Biotechnology, Santa Cruz, CA, USA) followed by enhanced chemiluminescence detection. Primary antibodies used in this study were anti-ATM (GeneScript, Piscataway, NJ, USA), anti-SMC1, anti-NBS1, anti-Chk1/2 (Santa Cruz Biotechnology), and anti-p53 (Cell Signaling Technology, Danvers, MA, USA). Also specific anti-phosphorylation antibodies were used against phospho-ATM (Ser1981, Cell Signaling), phospho-SMC1 (Ser966, Bethyl Laboratories, Montgomery, TX, USA), phospho-NBS1 (Ser345), phospho-Chk1 (Ser317), phospho-Chk2 (Thr38), and phospho-p53 (Ser15 and Ser20, Cell Signaling Technology). Anti-actin antibody (Santa Cruz Biotechnology) was used to validate protein amount.

Flow cytometric analysis for cell cycling arrest and apoptosis. Cells were exposed to different concentrations of DFO $(300 \mu \mathrm{M})$, NCS $(0.5 \mu \mathrm{g} / \mathrm{ml})$, or $\mathrm{HU}(3 \mathrm{mM})$ for $24 \mathrm{~h}$. Cell cycle arrest was assessed by ploidy analysis after DNA staining with PI as previously described. ${ }^{30}$ Briefly, cells were trypsinized, washed with cold PBS, and then fixed with ice-cold 70\% ethanol and precipitated for $2 \mathrm{~h}$ at ice. After fixation, cells were suspended in room temperature PBS, treated with RNaseA $(50 \mathrm{mg} / \mathrm{ml})$, and then incubated for $30 \mathrm{~min}$ at $37^{\circ} \mathrm{C}$ followed by stained with PI $(50 \mu \mathrm{g} / \mathrm{ml})$. Samples were analyzed using FACSCalibur 
flow cytometer (BD Biosciences, Franklin Lakes, NJ, USA). Apoptosis was determined by Annexin V-FITC Apoptosis Detection Kit (EMD Chemicals, Gibbstown, NJ, USA) according to manufacturer's protocol. In addition to antiannexin-FITC antibody, PI was used to distinguish between viable, early apoptotic and necrotic, or late-apoptotic cells. Chemical-induced apoptosis was determined as fold induction compared with that in mock-treated cells. The data were analyzed with CellQuestPro software (BD Biosciences).

Transfection. Transient transfection of both wild-type HCT 116 and HCT116ATR(-/flox) cells with a plasmid expressing Cre-GFP fusion protein was performed for $48 \mathrm{~h}$ with Lipofectamin 2000 (Invitrogen) according to manufacturer's instruction, followed by treatment with DFO or $\mathrm{HU}$ for $24 \mathrm{~h}$. Transfection efficiency was monitored by green fluorescent signals of Cre-GFP under the microscope. GFPnegative cells were excluded by gating with side scatter (SSC) and Green fluorescent channel (FL1), and then GFP-positive/apoptotic cells were analyzed by AnnexinV-APC Apoptosis detection kit (eBioscience, San Diego, CA, USA) and PI according to manufacturer's instruction.

Statistical analysis and densitometry. Data are shown as mean \pm S.D. of one of the representative experiment from two to three independent experiments. The significance of differences in the mean values was determined by the two-tailed unpaired Student's $t$-test. $P$-values $<0.05$ were considered to be statistically significant. The level of phosphorylation and expression of proteins were measured by densitometry using ImageJ software (National Institutes of Health) and the data were expressed as arbitrary units relative to band intensity of total protein or internal control.

\section{Conflict of interest}

The authors declare no conflict of interest.

Acknowledgements. We thank all the members of the Ouchi laboratory for discussion. We also thank Dr. B Vogelstein and Dr. E Hendrickson for sharing HCT116 isogenic cell line. These studies are supported by grants from the National Institutes of Health (RO1-CA79892 and RO1-CA90631) and grants from Susan G Komen Foundation and Avon Foundation.

1. Mahaney BL, Meek K, Lees-Miller SP. Repair of ionizing radiation-induced DNA doublestrand breaks by non-homologous end-joining. Biochem J 2009; 417: 639-650.

2. Lamarche BJ, Orazio NI, Weitzman MD. The MRN complex in double-strand break repair and telomere maintenance. FEBS Lett 2010; 584: 3682-3695.

3. Moynahan ME, Jasin M. Mitotic homologous recombination maintains genomic stability and suppresses tumorigenesis. Nat Rev Mol Cell Biol 2010; 11: 196-207.

4. Shiloh Y. ATM and related protein kinases: safeguarding genome integrity. Nat Rev Cancer 2003; 3: 155-168

5. Uziel T, Lerenthal Y, Moyal L, Andegeko Y, Mittelman L, Shiloh Y. Requirement of the MRN complex for ATM activation by DNA damage. EMBO J 2003; 22: 5612-5621.

6. Lin $Q$, Yun Z. Impact of the hypoxic tumor microenvironment on the regulation of cancer stem cell characteristics. Cancer Biol Thr 2010; 9: 949-956.

7. Yuan J, Narayanan L, Rockwell S, Glazer PM. Diminished DNA repair and elevated mutagenesis in mammalian cells exposed to hypoxia and low pH. Cancer Res 2000; 60: 4372-4376.

8. Bristow RG, Hill RP. Hypoxia and metabolism. Hypoxia, DNA repair and genetic instability. Nat Rev Cancer 2008; 8: 180-192.

9. Hammond EM, Denko NC, Dorie MJ, Abraham RT, Giaccia AJ. Hypoxia links ATR and p53 through replication arrest. Mol Cell Biol 2002; 22: 1834-1843.

10. Hammond EM, Dorie MJ, Giaccia AJ. ATR/ATM targets are phosphorylated by ATR in response to hypoxia and ATM in response to reoxygenation. J Biol Chem 2003; 278 : 12207-12213.

11. Bencokova Z, Kaufmann MR, Pires IM, Lecane PS, Giaccia AJ, Hammond EM. ATM activation and signaling under hypoxic conditions. Mol Cell Biol 2009; 29: 526-537.

12. Strunnikov AV, Jessberger R. Structural maintenance of chromosomes (SMC) proteins: conserved molecular properties for multiple biological functions. Eur J Biochem 1999; 263 6-13.

13. Watrin $\mathrm{E}$, Peters $\mathrm{JM}$. The cohesin complex is required for the DNA damage-induced G2/M checkpoint in mammalian cells. EMBO J 2009; 28: 2625-2635.

14. Kim ST, Xu B, Kastan MB. Involvement of the cohesin protein, Smc1, in ATM-dependent and independent responses to DNA damage. Genes Dev 2002; 16: 560-570.

15. Tomimatsu N, Mukherjee B, Burma S. Distinct roles of ATR and DNA-PKcs in triggering DNA damage responses in ATM-deficient cells. EMBO Rep 2009; 10: 629-635.

16. Kim BM, Choi JY, Kim YJ, Woo HD, Chung HW. Reoxygenation following hypoxia activates DNA-damage checkpoint signaling pathways that suppress cell-cycle progression in cultured human lymphocytes. FEBS Lett 2007; 581: 3005-3012.

17. Kim BM, Choi JY, Kim YJ, Woo HD, Chung HW. Desferrioxamine (DFX) has genotoxic effects on cultured human lymphocytes and induces the p53-mediated damage response. Toxicology 2007; 229: 226-235.

18. Bakkenist CJ, Kastan MB. DNA damage activates ATM through intermolecular autophosphorylation and dimer dissociation. Nature 2003; 421: 499-506.

19. Cliby WA, Roberts CJ, Cimprich KA, Stringer CM, Lamb JR, Schreiber SL et al. Overexpression of a kinase-inactive ATR protein causes sensitivity to DNA-damaging agents and defects in cell cycle checkpoints. EMBO J 1998; 17: 159-169.

20. Weterings $E$, Chen DJ. DNA-dependent protein kinase in nonhomologous end joining: a lock with multiple keys? J Cell Biol 2007; 179: 183-186.

21. Bunz F, Dutriaux A, Lengauer C, Waldman T, Zhou S, Brown JP et al. Requirement for p53 and p21 to sustain G2 arrest after DNA damage. Science 1998; 282: 1497-1501.

22. Cortez D, Guntuku S, Qin J, Elledge SJ. ATR and ATRIP: partners in checkpoint signaling Science 2001; 294: 1713-1716.

23. Ruis BL, Fattah KR, Hendrickson EA. The catalytic subunit of DNA-dependent protein kinase regulates proliferation, telomere length, and genomic stability in human somatic cells. Mol Cell Biol 2008; 28: 6182-6195.

24. Kim BM, Chung HW. Desferrioxamine (DFX) induces apoptosis through the p38-caspase8Bid-Bax pathway in PHA-stimulated human lymphocytes. Toxicol Appl Pharmacol 2008; 228: 24-31.

25. Ambrosini G, Nath AK, Sierra-Honigmann MR, Flores-Riveros J. Transcriptional activation of the human leptin gene in response to hypoxia. Involvement of hypoxia-inducible factor 1. J Biol Chem 2002; 277: 34601-34609.

26. Ertabak A, Kutluay T, Unlu A, Turkozkan N, Cimen B, Yaman H. The effect of desferrioxamine on peroxynitrite-induced oxidative damage in erythrocytes. Cell Biochem Funct 2004; 22: 149-152.

27. Yazdi PT, Wang Y, Zhao S, Patel N, Lee EY, Qin J. SMC1 is a downstream effector in the ATM/NBS1 branch of the human S-phase checkpoint. Genes Dev 2002; 16: 571-582.

28. Shang ZF, Huang B, Xu QZ, Zhang SM, Fan R, Liu XD et al. Inactivation of DNAdependent protein kinase leads to spindle disruption and mitotic catastrophe with attenuated checkpoint protein 2 phosphorylation in response to DNA damage. Cancer Res 2010; 70: 3657-3666.

29. Wong RW, Blobel G. Cohesin subunit SMC1 associates with mitotic microtubules at the spindle pole. Proc Natl Acad Sci USA 2008; 105: 15441-15445.

30. Ongusaha PP, Ouchi T, Kim KT, Nytko E, Kwak JC, Duda RB et al. BRCA1 shifts p53-mediated cellular outcomes towards irreversible growth arrest. Oncogene 2003; 22 $3749-3758$.

\section{(c)}

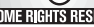

licensed under the Creative Commons Attribution-Noncommercial-No Derivative Works 3.0 Unported License. To view a copy of this license, visit http://creativecommons.org/licenses/by-nc-nd/3.0/ 